Case Report

\title{
Anorectal Gastrointestinal Stromal Tumor: A Case Report and Literature Review
}

\author{
Sanjeev Singhal, ${ }^{1}$ Anu Singhal, ${ }^{2}$ Rahul Tugnait, ${ }^{1}$ Vineet Varghese, ${ }^{1}$ Bishwanath Tiwari, \\ Pankaj K. Arora, ${ }^{1}$ Pawan Malik, ${ }^{1}$ Mriganka Deuri Bharali, ${ }^{1}$ Ankur Subhash Dhuria, ${ }^{1}$ \\ Pushkar Chauhan, ${ }^{1}$ Chandrakant Singh, ${ }^{1}$ Amit Ballani, ${ }^{3}$ and Vishnu Panwar ${ }^{4}$ \\ ${ }^{1}$ Department of Surgery, Northern Railway Central Hospital, New Delhi, India \\ ${ }^{2}$ Department of Radiology, ESI Model Hospital and PGIMSR, Basaidarapur, New Delhi 110001, India \\ ${ }^{3}$ Department of Radiology, Northern Railway Central Hospital, New Delhi, India \\ ${ }^{4}$ Department of Anaesthesia, Northern Railway Central Hospital, New Delhi, India
}

Correspondence should be addressed to Anu Singhal; drsinghal_anu@yahoo.co.in

Received 29 January 2013; Accepted 25 February 2013

Academic Editors: G. Bassotti, O. I. Giouleme, and Ö. Yönem

Copyright (C) 2013 Sanjeev Singhal et al. This is an open access article distributed under the Creative Commons Attribution License, which permits unrestricted use, distribution, and reproduction in any medium, provided the original work is properly cited.

Gastrointestinal stromal tumors or "GIST" are mesenchymal neoplasms expressing KIT(CD117) tyrosine kinase and showing the presence of activating mutations in KIT or PDGFR $\alpha$ (platelet-derived growth factor alpha). GIST of anal canal is an extremely rare tumor, accounting for only $3 \%$ of all anorectal mesenchymal tumors and $0.1-0.4 \%$ of all GIST. GIST with large tumor size and high mitotic activity are highly malignant, but the biological behavior of anorectal GIST is less clear. Abdominoperineal resection (APR) or conservative surgery is the best treatment option. Imatinib mesylate, a tyrosine kinase inhibitor, has shown promising results in its management. We present a case of anorectal GIST diagnosed by computed tomography (CT) scan, magnetic resonance imaging (MRI), and colonoscopy with biopsy. The patient underwent abdominoperineal resection (APR) and was confirmed on histopathology to have anal canal GIST with tumor size more than $5 \mathrm{~cm}$ in maximum dimension and mitotic figures more than $5 / 50$ high power field (HPF). The CD117-immunoreactive score-was 3+ in spindled cells. Therefore the patient was put on adjuvant imatinib mesylate $400 \mathrm{mg}$ daily.

\section{Introduction}

Gastrointestinal stromal tumor or "GIST" was a name given in 1983 to a group of gastrointestinal tumors which were otherwise unclassifiable as being of smooth muscle or neurogenic origin [1]. They are mesenchymal neoplasms expressing KIT(CD117) tyrosine kinase and showing presence of activating mutations in KIT or PDGFR $\alpha$ (platelet-derived growth factor alpha) [2]. It is the commonest gastrointestinal mesenchymal tumor [3] with the commonest site being stomach (50-60\%), followed by small intestine (30-40\%), colon (7\%), and oesophagus (1\%) [4]. GIST of anal canal and rectum are often grouped together and account for nearly $5 \%$ of all GIST $[4,5]$. However, of these only $2-8 \%$ are from anal canal, making GIST of anal canal an extremely rare tumor $[6,7]$.

\section{Case Report}

A 61-year-old male presented with pain during defecation and occasional bleeding per rectum over 2 months. Pain was nonradiating, dull aching, and persistent. Pain increased with constipation. Bleeding was frank red and came as drops after passage of stool. There was no tenesmus. There was no dizziness, weakness, pica, or weight loss. There was no significant relief with medication.

The patient was not a known case of piles/diabetes mellitus/hypertension/tuberculosis/or any other chronic ailment. The patient was a known alcoholic and smoker. He had no urinary complaints. There were no other complaints referable to chest and cardiac or nervous system.

He was a heterosexual with no known drug allergies.

On examination he was moderately built and of average nourishment. Karnofsky performance scale was more 


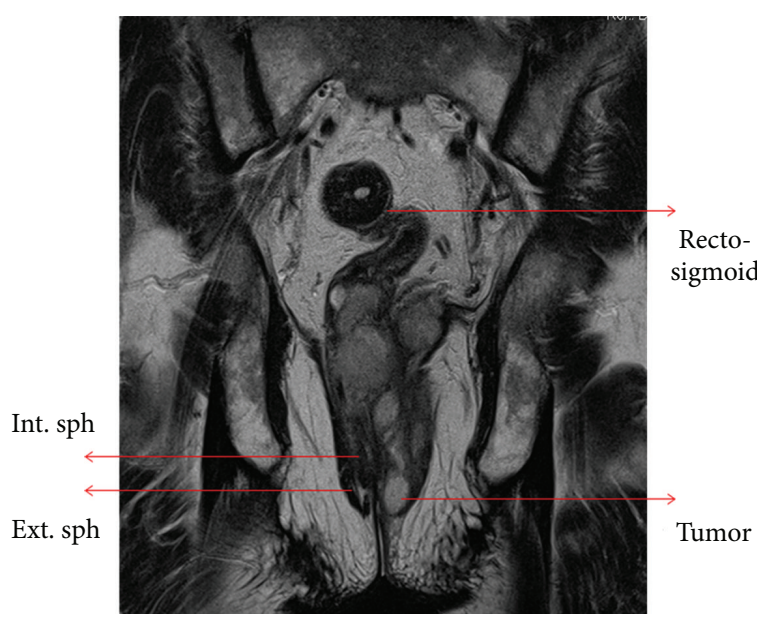

FIGURE 1: T2W sagittal section showing tumor extending up to anal verge and involving left wall of anal canal with loss of sphincteric anatomy.

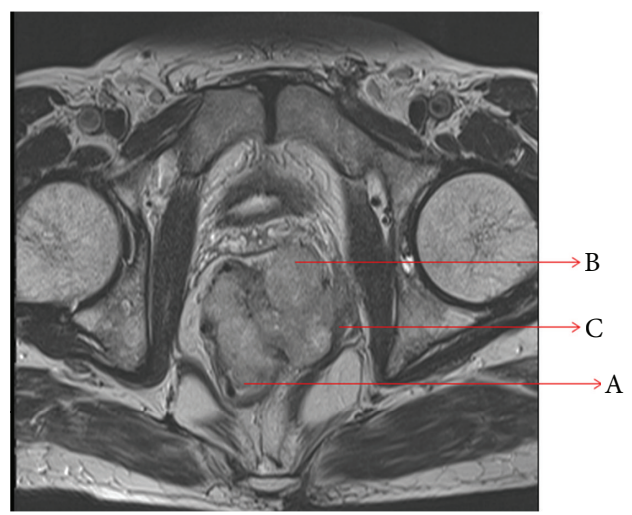

FIGURE 2: T2W axial image through distal rectum showing partly endophytic (A) and partly exophytic (B) mass having intermediate signal intensity. Left puborectalis muscle is invaded, thickened, and retracted $(\mathrm{C})$.

than 80 . There was no pallor/icterus/pedal edema/lymphadenopathy. On digital rectal examination, a hard indurated nontender mass was felt from 2 oclock to 9 o'clock, starting from the anal verge. The mass had a bosselated surface, and the overlying mucosa was tethered. The upper border of the mass could not be reached. No inguinal, iliac, para aortic, or supraclavicular lymph nodes were palpable.

His hematological and biochemical parameters were all within normal limits, and he was HIV seronegative. His transrectal ultrasound revealed an enlarged prostate with insignificant postvoid residual urine. There was significant illdefined mural thickness and hypervascularity along anorectal canal causing indentation of prostate. CT scan of the abdomen and pelvis showed an endo-exophytic soft tissue mass at anorectum suggestive of mitotic pathology. MRI pelvis revealed a lesion involving rectum and anal canal with extension into intersphincteric plane and puborectalis (Figures 1 and 2). His colonoscopy was suggestive of anorectal carcinoma, and colonoscopy guided biopsy was suggestive of

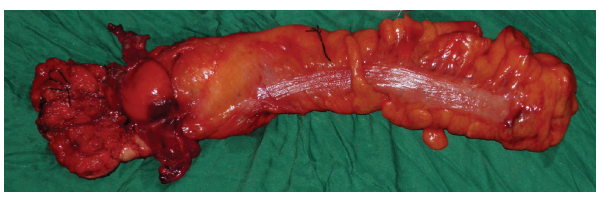

FIGURE 3: Abdominoperineal resection specimen.

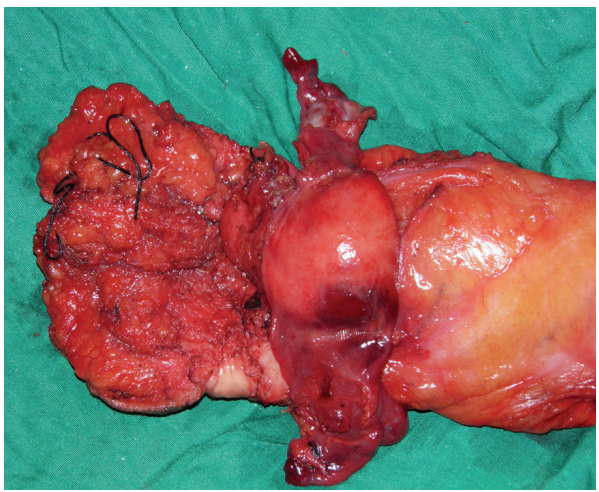

FIGURE 4: Growth in anorectal region.

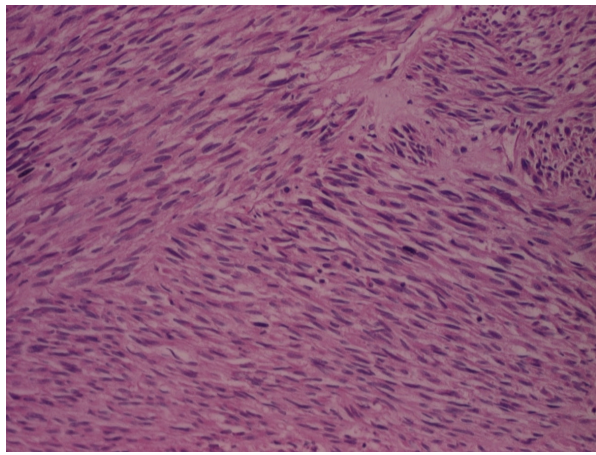

Figure 5: Spindle cells arranged in fascicles and showing mild nuclear pleomorphism and mitotic activity (100x magnification).

spindle cell carcinoma. A repeat punch biopsy was suggestive of leiomyosarcoma.

The patient underwent abdominoperineal resection with end colostomy (Figures 3 and 4). Histopathological examination report revealed anal canal GIST with tumor size more than $5 \mathrm{~cm}$ in maximum dimension. Mitotic figures were more than 5/50 HPF. Proximal and distal resected margins were uninvolved by tumor. Circumferential resected margin was less than $0.1 \mathrm{~cm}$ away from tumor. There was no lymphovascular invasion. Out of the 6 lymph nodes resected none were involved by tumor. The CD117-immunoreactive score-was $3+$ in spindled cells (Figures 5 and 6).

\section{Discussion}

The incidence of anal cancer in the western world is between 7 and 9 per million population. It contributes to only $1.5 \%$ of all malignancies of the digestive system [8]. Of these, GIST form only 3\% of anorectal mesenchymal tumors [7]. However, the exact data in eastern world may be different, as 


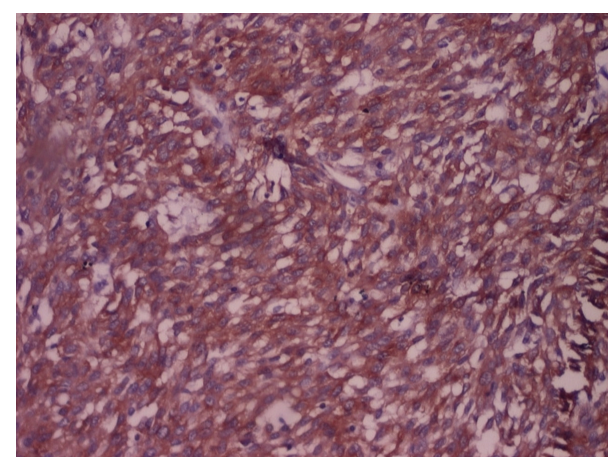

FIGURE 6: Spindle cells showing strong immunoreactivity for CD117 on IHC (score $3+$ to $4+$ ) (100x magnification).

in China, unlike in western countries, rectal cancer accounts for approximately $70 \%$ of colorectal cancers [9].

The anal canal extends from the perianal skin (anal verge) to the rectal mucosa. An important landmark within the canal is the dentate or pectinate line, which represents the end of the squamous mucosa and the beginning of a zone of transition from squamous to nonsquamous (either transitional or rectal glandular) mucosa. Thus, tumors arising in the anal canal can be either keratinizing or nonkeratinizing depending on their location in relation to the dentate line. Importantly, both keratinizing and nonkeratinizing tumors appear to have similar biology and prognosis [10]. Adenocarcinomas, on the other hand, behaves quite differently and should be treated like rectal cancers. Since there is no easily identifiable landmark between the rectum and anal canal, one has to rely on the pathologic classification of tumors in this area rather than the surgical or endoscopic classification [6].

GIST are currently thought to originate from interstitial cells of Cajal. The presence of interstitial Cajal-like cells has been reported in several extraintestinal organs including urinary bladder, prostate gallbladder, omentum, uterus, fallopian tube, and atrial and ventricular myocardium [11, 12]. This may explain the development of extraintestinal GIST $[13,14]$. Mutational statuses of c-KIT and PDGFR $\alpha$ genes are the basis for the diagnosis of this neoplasia and represent the criteria for surgical therapy, expected chemotherapy response, and clinical outcomes [6].

Risk factors for anorectal malignancies include female sex, patients with history of human papilloma virus (HPV) infection, human immunodeficiency virus- (HIV-) positive patients, patients who engage in anal receptive intercourse, presence of sexually transmitted disease, a history of more than 10 sexual partners, and a history of cervical, vulval, or vaginal cancers. Immunosuppressed individuals including renal transplant patients and those on chronic glucocorticoid therapy also appear to be at increased risk. Smoking is also a risk factor [6].

Most patients present with locoregional disease, and less than $20 \%$ of patients will present with or develop distant metastases. GIST with large tumor size and high mitotic activity are highly malignant, but the biological behavior of anorectal GIST is less clear [5, 15]. GIST of size $<2 \mathrm{~cm}$ and mitosis $<5$ per 50 HPF were indolent, whereas those with size
$>5 \mathrm{~cm}$ and/or mitosis $>5$ per $50 \mathrm{HPF}$ were highly malignant $[5,16]$. Changchien identified age $<50$ and size $>5 \mathrm{~cm}$ as independent prognostic markers [17].

GIST are best treated by surgery and are not radioor chemosensitive. However, controversy exists whether abdominoperineal resection (APR) or conservative surgery is the best alternative [15]. Though the incidence of local recurrence is lower after APR, the distant metastasis and survival are not significantly different [17]. Patterns of recurrence and metastasis for anorectal GIST are the same as for GIST elsewhere, and the disease usually has a long or protracted course. Therefore long followups are essential and local recurrences if any can be reoperated, if resectable [15]. As regards adjuvant or salvage therapy imatinib mesylate, a tyrosine kinase inhibitor, has shown promising results in the management of patients with GIST [14]. Tumor responses to imatinib are seen in $80 \%$ of patients. However, kinase inhibition by imatinib is not uniformly successful [3]. It has been suggested that low risk GIST with size $<2 \mathrm{~cm}$ and mitosis $<5$ per 50 HPF may be considered for local excision if sphincter saving surgery is technically feasible, and more aggressive GIST should be treated with radical excision [15].

Anorectal GIST, though rare, should be considered in the differential diagnosis of tumors in this region, especially if the pre-operative biopsy is equivocal. Gross and histopathological are both important, as prognosis depends on tumor size as well as grade. However, prognosis is usually better than for corresponding carcinomas in the region. Immunohistochemistry is a must, as CD-117 score is not only diagnostic but also guides adjuvant therapy and is an important prognostic marker.

Our case was a case of invasive anal canal GIST with tumor size more than $5 \mathrm{~cm}$ in maximum dimension. Mitotic figures were more than 5/50 HPF. The CD117-immunoreactive score was-3+ in spindled cells. Hence our patient has been put on adjuvant imatinib mesylate $400 \mathrm{mg}$ daily.

\section{Ethical Approval}

The study is cleared by the ethical committee of the hospital, and patients have given consent for the use of their clinical data for publication purpose.

\section{Conflict of Interests}

The authors declare that they do not have any conflict of interests.

\section{Authors' Contribution}

All authors have contributed significantly to the paper in terms of clinical material, radiological diagnosis, surgical craft, and final preparation of the paper.

\section{References}

[1] F. van der Aa, R. Sciot, W. Blyweert et al., "Gastrointestinal stromal tumor of the prostate," Urology, vol. 65, no. 2, p. 388, 2005. 
[2] M. C. Heinrich, C. D. Blanke, B. J. Druker, and C. L. Corless, "Inhibition of KIT tyrosine kinase activity: a novel molecular approach to the treatment of KIT-positive malignancies," Journal of Clinical Oncology, vol. 20, no. 6, pp. 1692-1703, 2002.

[3] A. Ghobadi, W. Kabbani, B. Barker, and J. E. Dowell, "Rectal GI stromal tumor mimicking a prostate mass," Journal of Clinical Oncology, vol. 25, no. 36, pp. 5827-5828, 2007.

[4] M. Miettinen, M. Sarlomo-Rikala, and J. Lasota, "Gastrointestinal stromal tumours," Annales Chirurgiae et Gynaecologiae, vol. 87, no. 4, pp. 278-281, 1998.

[5] M. Miettinen, M. Furlong, M. Sarlomo-Rikala, A. Burke, L. H. Sobin, and J. Lasota, "Gastrointestinal stromal tumors, intramural leiomyomas, and leiomyosarcomas in the rectum and anus: a clinicopathologic, immunohistochemical, and molecular genetic study of 144 cases," American Journal of Surgical Pathology, vol. 25, no. 9, pp. 1121-1133, 2001.

[6] G. R. Nigri, M. Dente, S. Valabrega et al., "Gastrointestinal stromal tumor of the anal canal: an unusual presentation," World Journal of Surgical Oncology, vol. 5, article 20, 2007.

[7] J. A. Tworek, J. R. Goldblum, S. W. Weiss, J. K. Greenson, and H. D. Appelman, "Stromal tumors of the anorectum: a clinicopathologic study of 22 cases," American Journal of Surgical Pathology, vol. 23, no. 8, pp. 946-954, 1999.

[8] J. C. Bendell and D. P. Ryan, "Current perspectives on anal cancer," Oncology, vol. 17, no. 4, pp. 492-503, 2003.

[9] D. B. Zhao, Y. K. Wu, Y. F. Shao, C. F. Wang, and J. Q. Cai, "Prognostic factors for 5-year survival after local excision of rectal cancer," World Journal of Gastroenterology, vol. 15, no. 10, pp. 1242-1245, 2009.

[10] H. E. Uronis and J. C. Bendell, "Anal cancer: an overview," Oncologist, vol. 12, no. 5, pp. 524-534, 2007.

[11] F. van der Aa, T. Roskams, W. Blyweert, and D. De Ridder, "Interstitial cells in the human prostate: a new therapeutic target?” Prostate, vol. 56, no. 4, pp. 250-255, 2003.

[12] K. W. Min and M. Leabu, "Interstitial cells of Cajal (ICC) and gastrointestinal stromal tumor (GIST): facts, speculations, and myths," Journal of Cellular and Molecular Medicine, vol. 10, no. 4, pp. 995-1013, 2006.

[13] B. D. Gun, M. O. Gun, and Z. Karamanoglu, "Primary stromal tumor of the omentum: report of a case," Surgery Today, vol. 36, no. 11, pp. 994-996, 2006.

[14] C. H. Lee, Y. H. Lin, H. Y. Lin, C. M. Lee, and J. S. Chu, "Gastrointestinal stromal tumor of the prostate: a case report and literature review," Human Pathology, vol. 37, no. 10, pp. 13611365, 2006.

[15] J. C.-M. Li, S. S.-M. Ng, A. W.-I. Lo, J. F.-Y. Lee, R. Y.-C. Yiu, and K.-L. Leung, "Outcome of radical excision of anorectal gastrointestinal stromal tumors in Hong Kong Chinese patients," Indian Journal of Gastroenterology, vol. 26, no. 1, pp. 33-35, 2007.

[16] M. Miettinen, M. Majidi, and J. Lasota, "Pathology and diagnostic criteria of gastrointestinal stromal tumors (GISTs): a review," European Journal of Cancer, vol. 38, pp. S39-S51, 2002.

[17] C. R. Changchien, M. C. Wu, W. S. Tasi et al., "Evaluation of prognosis for malignant rectal gastrointestinal stromal tumor by clinical parameters and immunohistochemical staining," Diseases of the Colon and Rectum, vol. 47, no. 11, pp. 1922-1929, 2004. 


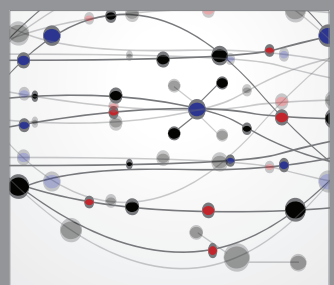

The Scientific World Journal
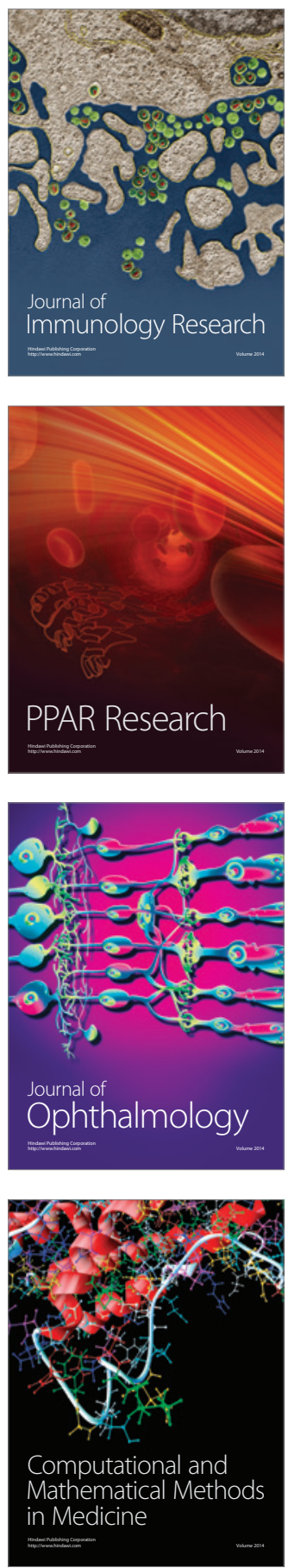

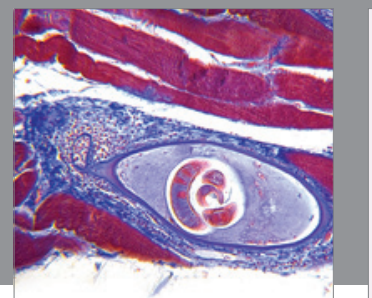

Gastroenterology

Research and Practice
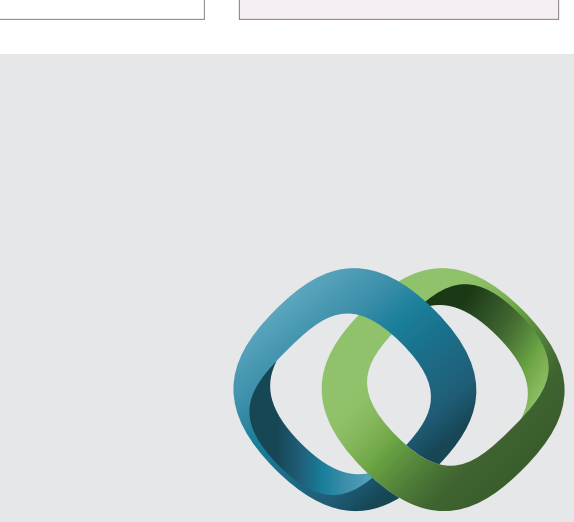

\section{Hindawi}

Submit your manuscripts at

http://www.hindawi.com
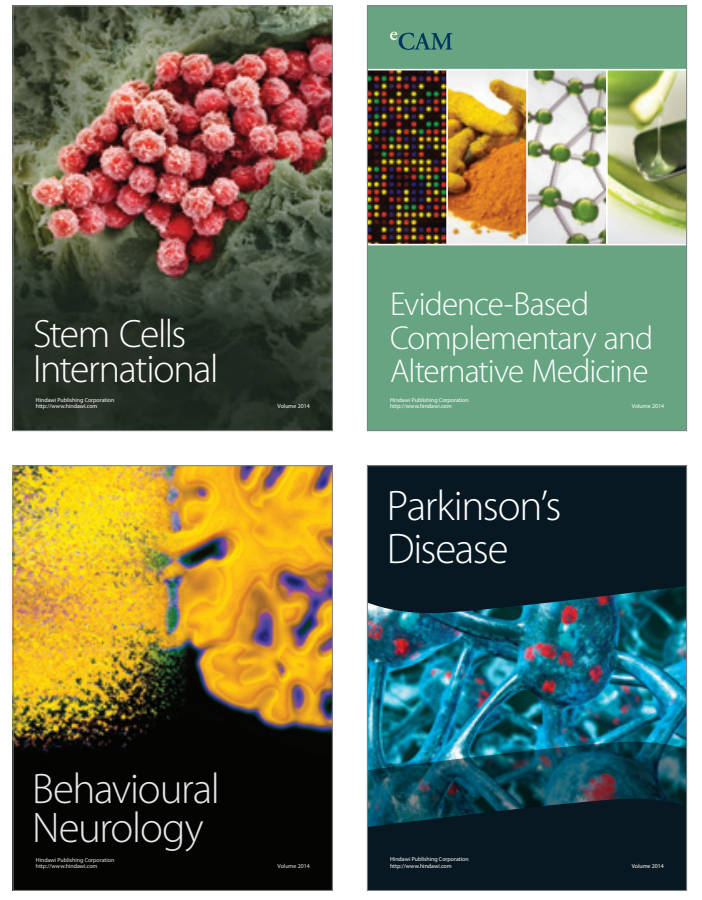
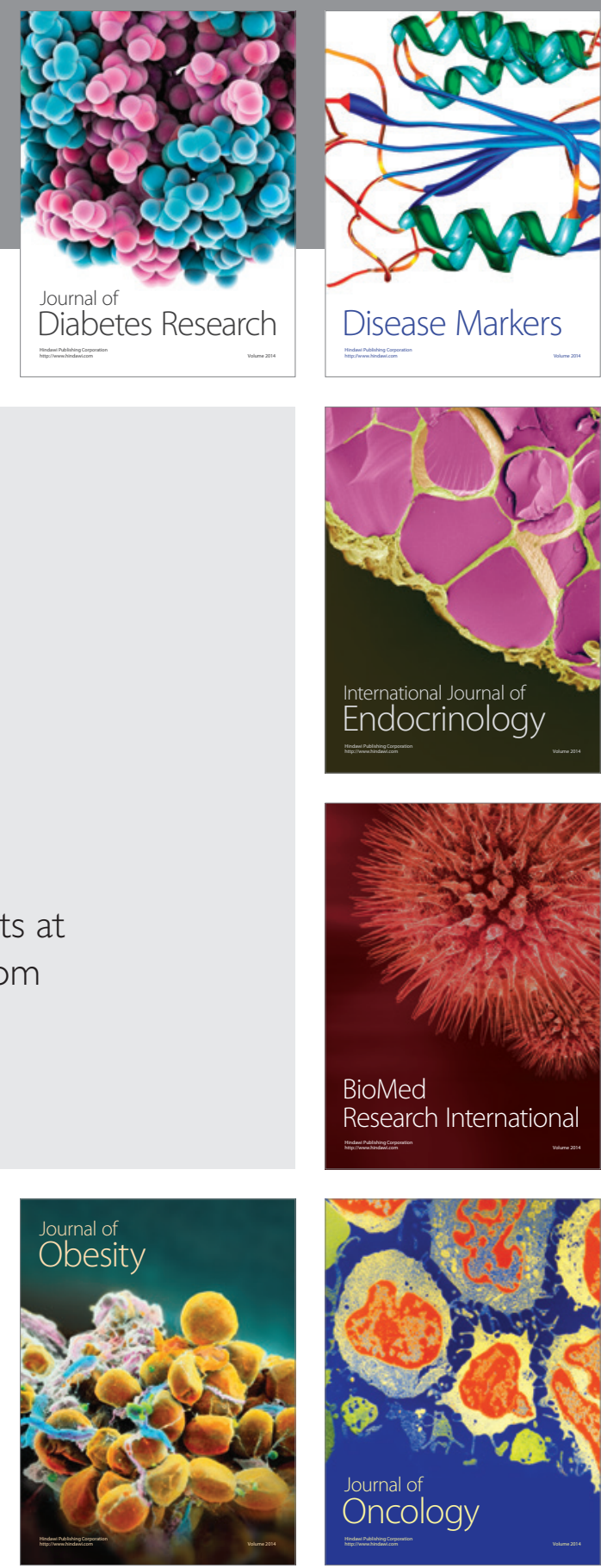

Disease Markers
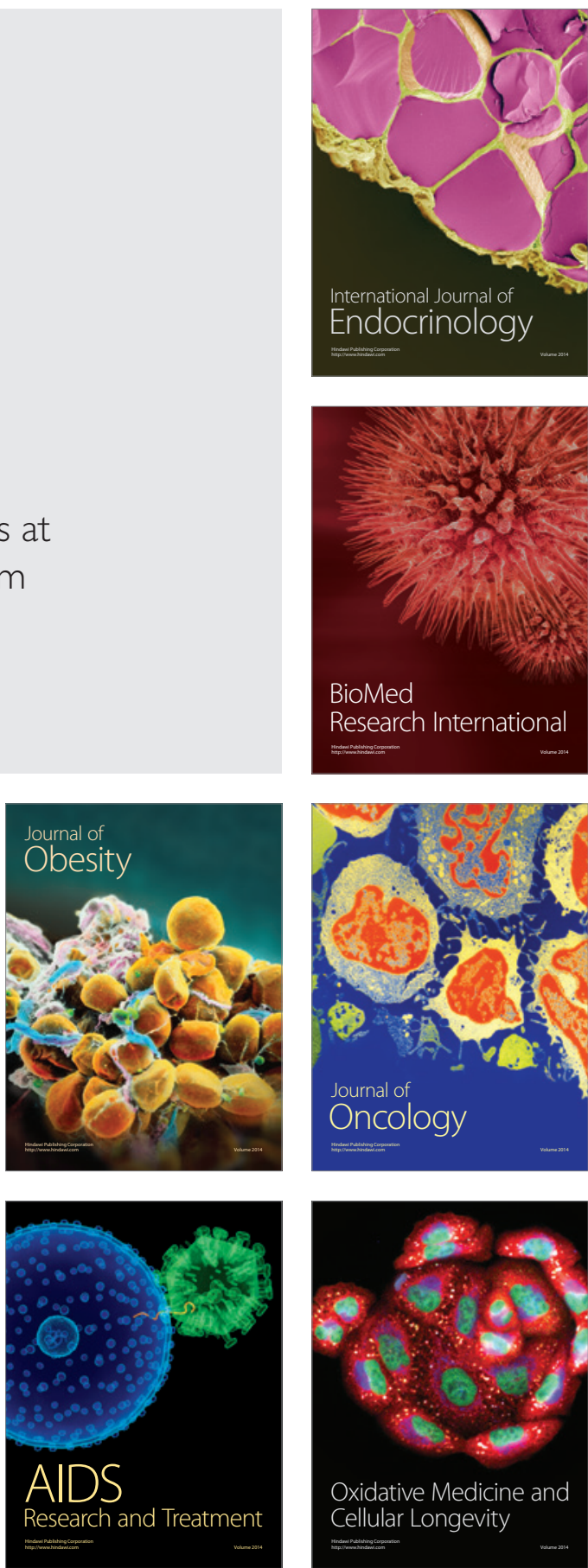